\title{
The Virtualization of Medical Education in response to COVID-19: A Harvard and McGill Pre-Clerkship Medical Student Perspective
}

\author{
Sara Al-Zubi, B.S. ${ }^{1}$ | Julianna Coleman, B.A., B.S. ${ }^{1}$ | Sarah Kordlouie, DEC ${ }^{2}$ | \\ Caroline Lee, B.A. $^{1}$ | Kaitlin Nuechterlein, B.Sc. ${ }^{2}$ | Feriel Rahmani, B.Sc. ${ }^{2}$ | Jamie E. \\ Shade, B.S. $^{1}$ | Zahra Talat, B.Sc. ${ }^{2}$ | Michael Teutenberg, B.Sc ${ }^{2}$ | Emily Wu, DEC ${ }^{2}$
}

\author{
${ }^{1}$ Harvard Medical School, Harvard \\ University, Boston, Massachusetts, United \\ States \\ ${ }^{2}$ McGill Faculty of Medicine, McGill \\ University, Montreal, Quebec, Canada

\section{Correspondence} \\ Kaitlin Nuechterlein \\ Email: kaitlin.nuechterlein@mail.mcgill.ca \\ Publication Date \\ October 30, 2020
}

MJM 2020 (18) 24

\section{McGill}

\section{Journal of Medicine}

www.mjmmed.com

\section{(i) (2)}

This work is licensed under a Creative Commons BY-NC-SA 4.0 International License.

\section{ABSTRACT}

In response to the spread of SARS-CoV-2 across North America in early March of 2020, Canadian and United States medical schools swiftly virtualized medical education for pre-clerkship students. With remote learning arrived novel challenges: barriers to students' comprehension of course material, difficulties conveying the nuances of patient interaction, and social hardships hindering students' continued progress. The 2020 Harvard-McGill Medical Student Exchange, a group of ten McGill University Faculty of Medicine and Harvard Medical School students, analyzed their institutions' respective responses in the virtualization of medical education and their personal experiences with remote pre-clerkship education. The authors' work provides insight into opportunities for mutual progress and cross-cultural exchange between Canadian and American medical schools, in the context of the COVID-19 pandemic. The authors detail potential changes to didactics, student research opportunities, support for students, and clerkship preparation that they expect would benefit pre-clerkship students in an ever-changing biomedical landscape. With gratitude toward their respective programs for their efforts in transitioning to virtual learning, the authors look toward a future of medical education increasingly interwoven with digital technology and responsive to social change.

\section{KEYWORDS}

Medical Education, COVID-19 Pandemic, International Perspectives 


\section{1 | PRELUDE}

The McGill-Harvard Exchange program, established by McGill alumnus and Harvard Medical School Assistant Professor Jeffery Semaan, gives select, pre-clerkship medical students from each institution the ability to collaborate with our medical colleagues across borders. Together, we are immersed in each city's hospital settings and experience world-class lectures from our respective institutions, reflecting on the similarities and differences of American and Canadian healthcare systems and medical education. With the dawn of the COVID-19 pandemic, our own exchange was postponed. However, the collaborative spirit of the participating students inspired an analysis of the virtualization of medical education from the unique perspective of first- and second-year Harvard and McGill medical students in the wake of the COVID-19 pandemic. In this paper, we detail changes to our respective institutions' pre-clerkship curriculum and important lessons learned from this process.

\section{2 | MEDICAL EDUCATION CUR- RICULUM}

Medical education in North America has perpetually undergone reformation to meet the needs of medical learners and the communities they serve. Recently, the rise of COVID-19 has been a source of change as restrictions disrupt medical education delivery. To understand how COVID-19 has impacted medical education it is important to understand the state of medical education prior to the pandemic.

\section{1 | A Brief History of Medical Education}

The expansion and exchange of scientific knowledge across cultures and centuries have shaped the core principles of Western medical education. The significance of physician involvement in medical education was first emphasized in the $5^{\text {th }}$ century BCE by the Hindu physician Charaka, who described the physician-pupil rela- tionship as crucial in developing clinical competency and genuine learning.(1) This relationship was further elaborated upon by Greek philosopher Plato and the Greek medical education system, as medical education was seen to be based more on experience with books only serving as adjuncts, never as replacements to practical experience.(1) As the Greek system of medical education began to gain popularity in Europe through crosscultural exchanges of knowledge, the first formal medical schools began to appear in the late Middle Ages in Northern Italy with two types of medical practitioners: academic doctors for which training was exclusively theoretical and practically-trained surgeons. $(2,3)$ Meanwhile, during the Islamic Golden Age $\left(8^{\text {th }}\right.$ to $13^{\text {th }}$ centuries $(E)$, medical education consisted of initial training in basic sciences followed by clinical training in hospitals, a model echoing most current medical curricula.(4)

The advent of the Western world's modern medical education system in the late $19^{\text {th }}$ century is largely the product of Sir William Osler's dedication to the advancement of scientific and practical medicine. Osler insisted on the importance of clinical exposure for medical student education through the implementation of bedside medicine, clinical clerkship, and residency programs that emphasized the value of patient interaction.(5) Prior to this point, a majority of medical student education was didactic, classroom-based teachings. Osler's reforms to medical education persist today, as bedside medicine remains one of the most important modalities in teaching clinical competency and has been shown to improve clinical diagnostic skills in medical students and residents.(6) However, this movement to bring medical students out of the lecture hall to the bedside is threatened by the emergence of pandemics-the most recent of which being COVID-19.

\section{2 | Medical Education's Adaptation to Previous Pandemics}

The impact of similar pandemics, such as Severe Acute Respiratory Syndrome Coronavirus 1 (SARS-CoV), on the education of medical students have been poorly explored. Conditions of uncertainty, limited resources 
and the need for reduced exposure rates in healthcare systems present necessary restrictions on the practice of bedside medicine training and clinical exposure yet threaten the quality of medical education.

In 2003, severe acute respiratory syndrome coronavirus 1 (SARS-CoV) threatened to become a global pandemic. Medical students in their clinical years of training experienced condensed or omitted clerkship rotations, impinged specialized clinical electives and delays in residency matches.(7) For pre-clerkship medical students, these clinical restrictions presented a new challenge of continuing to prepare for clinical training in the absence of patient contact. Didactic lectures became reinstated as the main focus for medical education and were only supplemented by simulated patients at many medical schools.(8) The long-term implications of these temporary restrictions on the quality of medical education during the SARS-CoV outbreak did not warrant extensive consideration as clinical teachings largely resumed after a relatively brief six-week cessation.(9) In contrast, with the COVID-19 pandemic measures and clinical restrictions on medical education expected to extend into the 2020-2021 academic year, the implications of reduced clinical exposure on the future generation of physicians can no longer be ignored.

\section{3 | Pre-Pandemic Medical Education at Harvard and McGill}

The pre-clerkship curricula at Harvard and McGill accomplish the same broad objective of preparing students for their clinical clerkships. However, there are key curricular differences that should be noted to truly understand the pandemic's unique impact on both schools, their respective faculties and student populations. To begin to explore the impact COVID-19 and the transition to a virtual platform has had on Harvard and McGill medical students, it is important to understand the current curriculum as put forth by both respective faculties.

Harvard University's "Pathways" curriculum is notable for its accelerated (14-month) pre-clerkship timeline. It emphasizes interactive case-based learning in groups of four students and minimizes didactic lectures. The coursework involves an integrated approach to physiology and pathophysiology that groups organ systems. Lastly, students participate in weekly clinical sessions during which we practice history-taking, physical examination, clinical reasoning, and seeing patients at a primary care clinic under the guidance of a faculty preceptor.(10)

The first 18 months of McGill University's preclerkship medical curriculum are dense with lectures, small group case-based activities, simulated clinical activities, and anatomy labs. This portion of the curriculum, known as Fundamentals of Medicine and Dentistry (FMD), is systems-based. The remainder of the preclerkship education is completed in Transition to Clinical Practice (TCP), which places medical students in a primarily clinical learning environment across various medical disciplines. During TCP, students are introduced to the tasks of clerkship and continue to have clinically focused lectures and assignments.(12,13)

The pre-clerkship years in medical education are foundational for clerkship experience and learning: knowledge acquired from didactic instruction can be translated to patient encounters and hospital-based learning. Textbook cases are further elaborated with new dimensions for the learner: patient-physician rapport, physical exam maneuvers and findings, operating hospital systems, resource considerations, and interprofessional work dynamics. In preparation for successful learning in the clinical environment, delivery of a wellrounded and thorough pre-clerkship curriculum is essential. With the disruption in accessibility to many of these in-person and hands-on learning activities during the pandemic, pre-clerkship learning has been compromised in preparing students for our clinical duties with patients and in the hospital.

Elements of pre-clerkship learning were differentially affected at Harvard and McGill given the different temporal organization of the curricula and the need to transition to remote learning. Hence, each university experienced unique challenges in adapting to certain inperson educational sessions. 


\section{3 | THE IMPACT OF COVID-19 ON MEDICAL EDUCATION}

The COVID-19 pandemic and subsequent shelter-athome measures have resulted in drastic changes to the delivery of medical education. These shifts may greatly impact how current and future pre-clerkship students learn course content and clinical skills needed for clerkships.

\section{1 | Class Lectures and Case Conferences}

With the onset of government-driven quarantine measures came the need for a new classroom medium: Zoom video conferencing, a product of Zoom Video Communications, Inc. For pre-clerkship McGill students, the lecture-intensive education was converted to online Zoom sessions, with a potentially greater opportunity for us to ask questions through the platform. However, small group clinical education had a less-optimal online transition, as it was replaced by large Zoom sessions, inheriting a more didactic nature. In this way, we were neither able to exercise our problem-solving skills when faced with clinical vignettes nor able to develop rapport with our colleagues for a smoother transition to clerkship.

Additionally, McGill first-year students were faced with the challenge of learning musculoskeletal anatomy remotely in the absence of in-person cadaver manipulation. Guided dissections of donated cadavers during in-person lab sessions suddenly became replaced with anatomist-led dissection videos and virtual anatomy applications for studying. Furthermore, physical exam techniques were replaced with descriptive handouts, video links, and live Zoom demonstrations led by professors and simulated patients. Remote anatomy and physical exam education was an extreme adjustment for both students and faculty alike. Under pandemic circumstances, the best possible accommodations were given; however, the virtual substitute truly highlighted the need for hands-on manual manipulation in anatomy education as virtual platforms were ill adapted to expose students to pertinent physiological variants.

When Harvard Medical School (HMS) pre-clerkship students transitioned to online learning, we were midway through the integrated physiology and pathophysiology course that covers cardiology, hematology, and pulmonology. In the abrupt shift to online learning, classes that had initially taken place in forty-student "learning studios" in which students collaborated in groups of four began taking place in one large Zoom session of 160 students. In this format, it was difficult to gain the level of engagement between faculty and students achieved in a smaller classroom setting. While faculty attempted to preserve the collaborative nature of the curriculum with the "Breakout Rooms" feature on Zoom, this feature in the large group did not replicate the face-to-face collaboration of in-person sessions. Before switching to virtual learning, we worked with the same group for 3-4 consecutive weeks and established strong team dynamics. However, the Zoom Breakout Rooms were randomized in each session, and we were working with classmates from other learning studios. While this format gave us the opportunity to collaborate with classmates whom we did not know as well, it was difficult for us to effectively work through cases with a different group of students each day. In addition, we could no longer work on team dynamics with a consistent group of 3-4 classmates over the course of several sessions together.

Faculty promptly responded to student feedback and adapted the technology to enable sessions to take place in the familiar setting of our forty-student classrooms. We still worked with a randomized "Breakout Group" instead of a consistent group. However, we could more effectively work through cases because we had had prior experience working with students from our learning studios. This smaller setting also allowed for greater student-faculty engagement.

\section{2 | COVID-19 Specific Education}

As it stands, COVID-19 will surely mark the medical education of current pre-clerkship students across the world. While the understanding of COVID-19 contin- 
ues to evolve on the merit of new scientific findings, there is a heightened need for greater COVID-specific pre-clerkship education to instruct students on proper safety protocols and best practices before the transition into a clinical environment with COVID-19.

In response to the pandemic, a team of students at Harvard Medical School quickly convened to create a COVID-19 Student Response Team to develop a structure that can adapt and address immediate COVID-19 student educational needs with the assistance of Harvard leadership and faculty. Part of this effort included the creation of COVID-19 curriculum modules and supplementary materials. In preparation for the clinical clerkships, second-year students are required to complete four of the eight modules on pathophysiology, communication of information about COVID-19, preparation for clinical roles, and medical ethics.

Serendipitously, McGill Medicine's first-year class had its courses on immunology and infectious diseases align with the onset of pandemic isolation measures. To supplement these topical subjects, some classes were replaced with COVID-19-related content. Lectures regarding virology, pharmacological trials, and transmission of COVID-19 were all tested on exams, bringing real-world relevance to the curriculum. We also received a lecture detailing the pandemic's COVID vs non-COVID clinic designations in the Province of Quebec, and how patients can access primary care through telemedicine consultations.

In order to give students additional time to explore new COVID classes, the medical curriculum should adjust to include these classes or decrease other workload to allow for proper exploration and understanding. Beyond the lecture-setting, clinical sessions on PPE use, proper hygiene, and COVID-19 protocols in both hospital and primary care settings will be vital to the safety of clerks and patients alike as the pandemic persists.

Furthermore, the limitations in our respective healthcare systems highlight the need for bioethics education while responding to the pandemic. As part of the HMS Essentials course curriculum, we had eight classes on Medical Ethics and Professionalism. These included discussions about rationing and futility in times of scarcity, principles of bedside rationing, ethics of disparities, and responsibilities of physicians to address patients' inequities and inequalities. McGill students likewise cover these subjects in their Medical Ethics and Health Law course, taught in second year, prior to clerkship. Yet, the need for more required ethics courses is evident, now more than ever, especially when COVID19 has exacerbated the existing disparities experienced within society. Black Americans are and continue to be disproportionately impacted by COVID-19 as shown by the infection and death rate.(15) Similar disparities have been observed in Canada's Indigenous communities, though Indigenous Services Canada (ISC) has failed to provide an accurate picture of the burden of disease nationwide for Indigenous peoples.(16) The current disparities highlight the importance of bioethics and the need for heightened ethics training in medical education to prepare future physicians to adequately address these disparities when encountered in clinic.

\section{3 | Considerations for Medical Student Research}

Pre-clerkship years are often an opportune time for medical students to conduct original research and produce publications, while acquiring skills in scientific inquiry and communication. In response to the spread of SARS-CoV-2, laboratories at McGill and Harvard were largely shuttered beginning in mid-March. Among the many medical students affected, Harvard's clinicianscientists in training might have faced the greatest repercussions of the ensuing hiatus in research. The laboratory ramp-down coincided with nearly three months of dedicated research time in the pre-clerkship curriculum for most MD-PhD students, impinging on a critical period for students' exploration of research interests prior to beginning doctoral work. To miss this opportunity might have notable implications for students with otherwise limited access to mentorship in laboratory-based research, especially those underrepresented in science.

In order to accommodate social-distancing guidelines, physician-scientists in training have redesigned their research rotations. Some students have planned 
revised laboratory experiments not requiring in-person training from other laboratory members. Others have opted for rotations involving computational research in fields as diverse as software engineering and public health studies. Such endeavours have allowed these students to work remotely without placing themselves or others at risk of infection. We surmise that the shift away from bench research might reveal novel opportunities for medical students' engagement in computational work, especially as artificial intelligence and automation begin their inevitable integration into clinical practice. Exposing clinician-scientists in training to this work might prove fruitful as knowledge of computational techniques becomes increasingly valuable in medicine.

\section{4 | CONCLUSION}

The emergence of COVID-19 marks an unprecedented disruption to medical education requiring intense and prompt attention from educators in both Canada and the United States. While the response from McGill and Harvard faculty and medical students alike has been to rise to the monumental challenge of a virtualized medical education, the reversion to didactic lectures has reiterated the historical narrative of in-person bedside teachings as imperative to medical students' clinical competency in the pre-clerkship years of training. These obligatory adaptations to the medical curriculum have emphasized the need for in-person components in anatomy education that allow manual manipulation to appreciate structural orientation, a heightened need for greater COVID-specific education to better prepare medical students for an eventual reintroduction into the clinical environment, and a greater need for ethics courses to shed light on the disproportional impact such pandemics have on vulnerable populations. Nevertheless, COVID-19 has taught a valuable lesson to faculty and medical students, highlighting the need to be agile in an ever-changing world. The profound effects of COVID-19 may forever change the approach to medical education for future physicians.

\section{REFERENCES}

1. Fulton JF. History of Medical Education. The BMJ 1953. https://www.bmj.com/content/2/4834/457 (accessed August 22, 2020).

2. Custers EJ, Cate OT. The History of Medical Education in Europe and the United States, With Respect to Time and Proficiency. Academic Medicine 2018;93. doi:10.1097/acm.0000000000002079.

3. Emmanouil P, Pavlos M, Avgerinos ED, Sofia A, Christos T. Evolution of medical education in ancient Greece. Chinese Medical Journal 2008;121:2202-6. doi:10.1097/00029330-20081101000018.

4. Falagas ME, Zarkadoulia EA, Samonis G. Arab science in the golden age (750-1258 C.E.) and today. The FASEB Journal 2006;20:1581-6. doi:10.1096/fj.06-0803ufm.

5. https://www.citationmachine.net/bibliographies/ebfc5c85737f-4417-ac17-e8c9c4f947e6

6. Peters $M$, Cate OT. Bedside teaching in medical education: a literature review. Perspectives on Medical Education 2013;3:76-88. doi:10.1007/s40037-013-0083-y.

7. Sherbino J, Atzema C. "SARS-Ed": severe acute respiratory syndrome and the impact on medical education. Annals of Emergency Medicine 2004;44:229-31. doi:10.1016/j.annemergmed.2004.05.021.

8. Clark J. Fear of SARS thwarts medical education in Toronto. Bmj 2003;326:784c-784. doi:10.1136/bmj.326.7393.784/c.

9. Ahmed $\mathrm{H}$, Allaf $\mathrm{M}$, Elghazaly $\mathrm{H}$. COVID-19 and medical education. The Lancet Infectious Diseases 2020;20:777-8. doi:10.1016/s1473-3099(20)30226-7.

10. Schwartzstein RM, Dienstag JL, King RW, Chang BS, Flanagan JG, Besche HC, et al. The Harvard Medical School Pathways Curriculum. Academic Medicine 2020; Publish Ahead of Print. doi:10.1097/acm.0000000000003270.

11. Pathways Curriculum Map 2020. https://meded.hms.harvard.edu/files/hms-meded/files/pathways_curriculum_map.pdf.

12. Curricular Innovations. Undergraduate Medical Education 2016. https://www.mcgill.ca/ugme/mdcm-curriculum-jointprograms/curricular-innovations (accessed August 22, 2020).

13. Program Learning Objectives. Undergraduate Medical Education 2018. https://www.mcgill.ca/ugme/mdcm-curriculumjoint-programs/curriculum-structure/program-learning-outcomes (accessed August 22, 2020).

14. M.D. C.M Program: Curriculum Schema 2019. https://www.mcgill.ca/ugme/files/ugme/mdcm_schema_2020-0207_en.pdf.

15. Price-Haywood EG, Burton J, Fort D, Seoane L. Hospitalization and Mortality among Black Patients and White Patients with Covid-19. New England Journal of Medicine 2020;382:2534-43. doi:10.1056/nejmsa2011686. 
16. Over 450 Indigenous COVID-19 cases across Canada and 7 deaths, reports Yellowhead Institute | CBC News. CBCnews 2020. https://www.cbc.ca/news/indigenous/yellowhead-institutecoronavirus-report-1.5565954 (accessed August 22, 2020). 


\section{Figures}

Pathways Curriculum Map

HARVARD MEDICAL SCHOOL
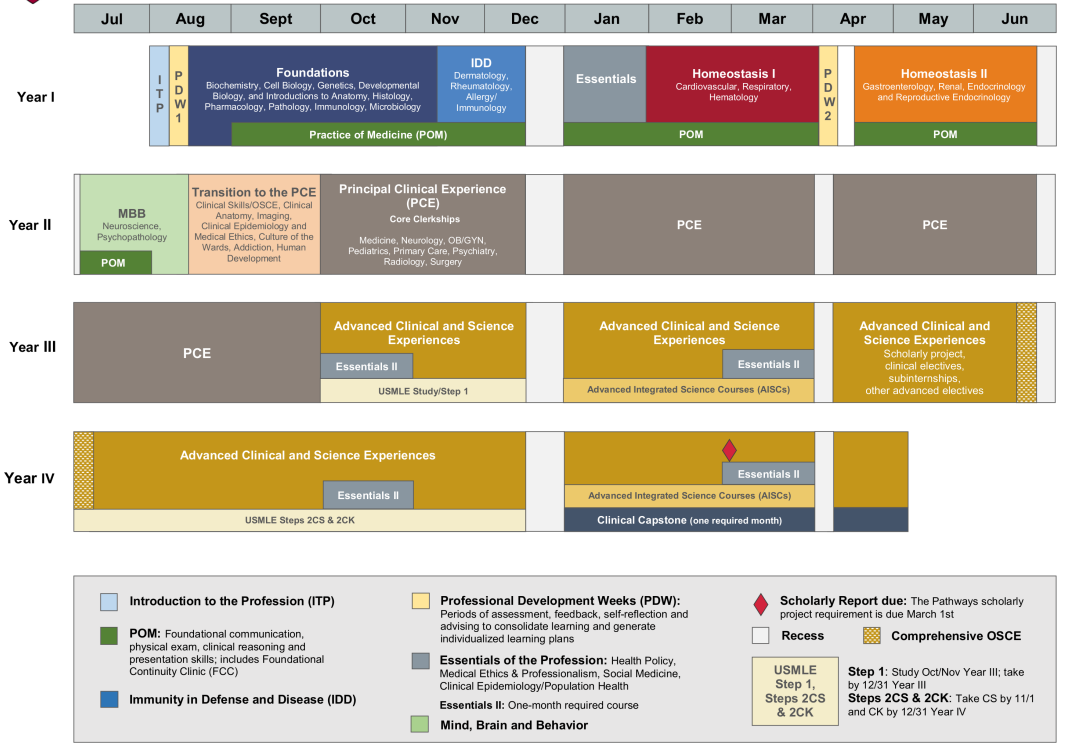

FIGURE 1 Visual schematic of Harvard Medical School's four-year "Pathways" curriculum. Pre-clerkship: Practice of Medicine (POM). Clinical: Transition to PCE, Principal Clinical Experience (PCE), Advanced Clinical and Sciences Experiences (11).

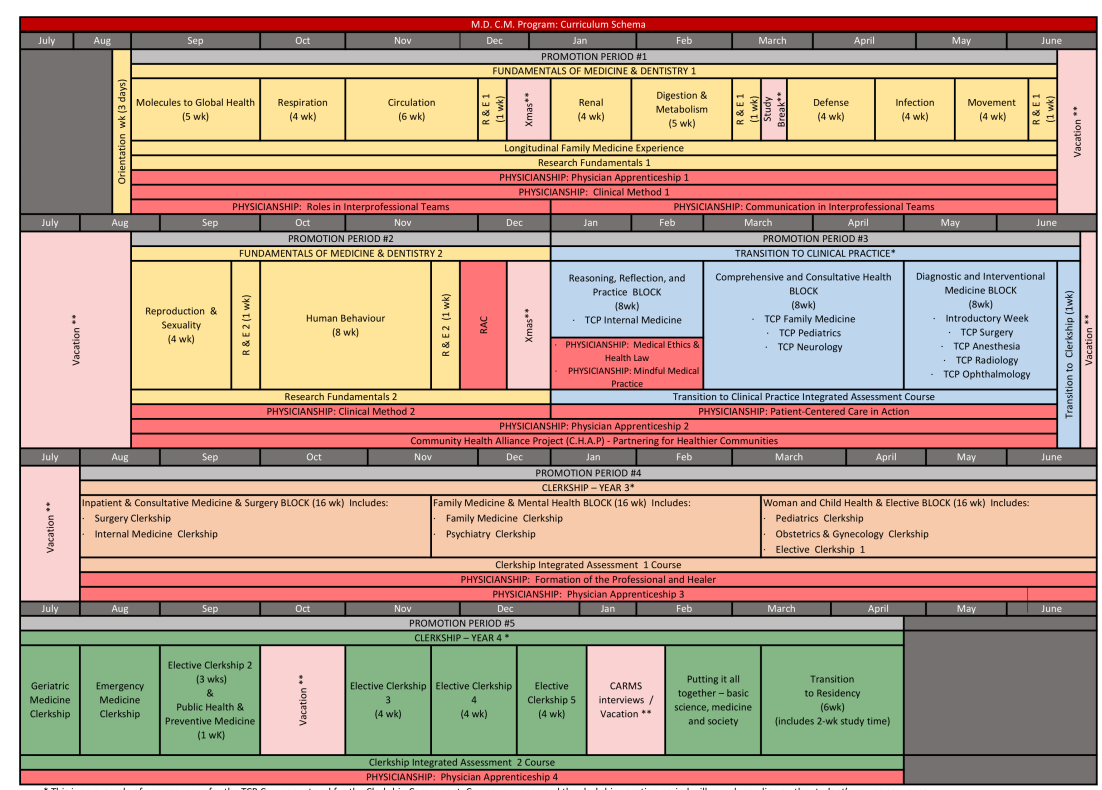

FIG URE 2 Visual schematic of McGill Medical School's four-year curriculum. Pre-clerkship: Fundamentals of Medicine and Dentistry (FMD) 1, 2. Clinical: Transition to Clinical Practice (TCP), Clerkship (14). 